\title{
Prevalence and outcome of isoniazid-monoresistant tuberculosis at a university hospital in Saudi Arabia
}

\author{
Khalifa M. Binkhamis, MBBS, FRCPC, Mohammed A. Bahatheg, Medical Student, Faisal A. Altahan, Medical Student, \\ Saleh S. Alwakeel, Medical Student, Khalid A. Almutairi, Medical Student, Abdullah F. Alsaeed, Medical Student, Dalal A. Alkadi, \\ MBBS, Hanan A. Alshafai, MBBS, Fahad M. Almajid, MBBS, FRCPC, Mazin A. Barry, MBBS. FRCPC.
}

\begin{abstract}
الأهداف: دراسة مدى انتشار مرض السل المقاوم لدواء الأيزونيازد، ونتائج

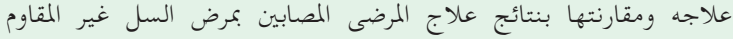
لالأيزونيازد.

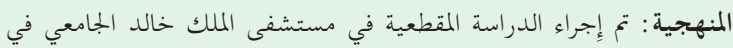

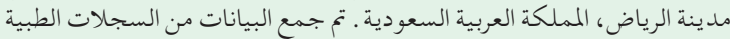

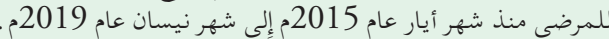

النتائج : وجدنا 105 مرضى مصابين بالسل . تسعة 80.6 8.6 من المرضى مصابين

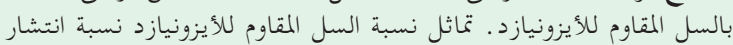

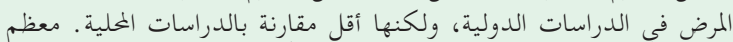

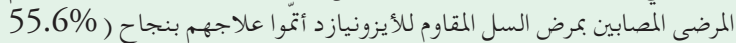

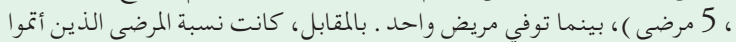

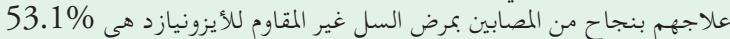

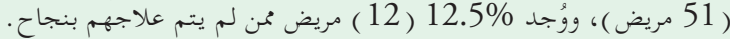

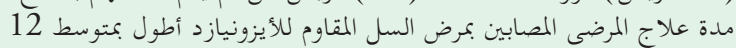

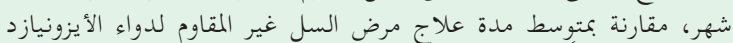

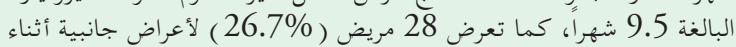

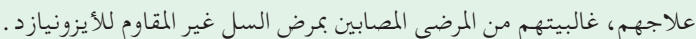

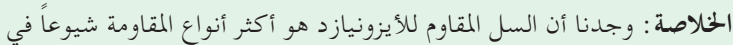

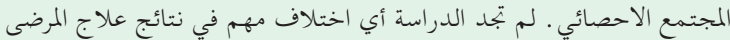

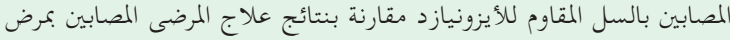

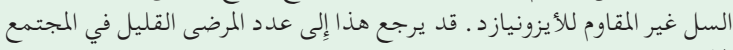

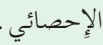

Objectives: To determine the prevalence and outcome in patients with isoniazid-monoresistant Mycobacterium tuberculosis complex and compare them to those in patients with non-isoniazid-monoresistant Mycobacterium tuberculosis.

Methods: This cross-sectional analytical study was conducted at King Khalid University Hospital, Riyadh, Saudi Arabia. The data were retrospectively collected from the electronic medical records of patients who tested positive for Mycobacterium tuberculosis between May 2015 and April 2019.
Results: We identified 105 patients infected with Mycobacterium tuberculosis. The prevalence proportion of isoniazid-monoresistant tuberculosis was 8.6\% $(\mathrm{n}=9)$. Five patients with isoniazid-monoresistant tuberculosis $(55.6 \%)$ were successfully treated, while one patient died. In the nonresistant population, 51 $(53.1 \%)$ patients were successfully treated. However, $12(12.5 \%)$ patients with no isoniazid resistance had an unsuccessful treatment outcome. The resistant group had a longer treatment duration with a mean of 12 months compared to the non-isoniazid-resistant group, with a mean treatment duration of 9.5 months. Twenty-eight patients (26.7\%) had adverse events, with the majority of them being in the non-isoniazidresistant group.

Conclusion: Isoniazid monoresistance is the most common form of drug resistance found in our population. Our study has not shown any significance in the outcome of isoniazid-resistant cases compared to non-isoniazid-resistant cases. This may be due to the low number of isoniazid-monoresistant cases in our population.

Keywords: Mycobacterium tuberculosis, isoniazid, drug resistance, prevalence, patient outcome assessment

Saudi Med J 2021; Vol. 42 (6): 636-642 doi: 10.15537/smj.2021.42.6.20200832

From the Department of Pathology (Binkhamis, Alkadi, Alshafai), College of Medicine, King Saud University, Riyadh, Kingdom of Saudi Arabia; from the Infectious Diseases Unit, Department of Medicine (Almajid, Barry), College of Medicine, King Saud University, Riyadh, Kingdom of Saudi Arabia; from King Saud University Medical City (Binkhamis, Alkadi, Alshafai, Almajid, Barry), King Saud University, Riyadh, Kingdom of Saudi Arabia; from the College of Medicine (Bahatheg, Altahan, Alwakeel, Almutairi, Alsaeed), King Saud University, Riyadh, Kingdom of Saudi Arabia.

Received 31st December 2020. Accepted 19th March 2021.

Address correspondence and reprint request to: Dr. Khalifa $M$. Binkhamis, Department of Pathology, College of Medicine, King Saud University. E-mail: kbinkhamis@ksu.edu.sa

ORCHID ID: https://orcid.org/0000-0002-5425-9531 
A mong the biggest consequential public health concerns worldwide is tuberculosis (TB). The global economic burden of $\mathrm{TB}$ from prevention to diagnosis and treatment is still increasing and has doubled since 2006. ${ }^{1}$ The latest World Health Organization (WHO) $\mathrm{TB}$ report has shown that 119 low and medium-income countries which constitute $97 \%$ of cases worldwide had funding that amounted to 6.8 billion USD in 2019. However, even these efforts consist only $67 \%$ of that 10.1 billion USD required in the global plan to end TB. ${ }^{1}$

Approximately, 10 million individuals globally had $\mathrm{TB}$ in 2018. The burden of the disease significantly varies among countries, ranging from less than 5 to more than 500 new cases per 100,000 population annually, with a global average of approximately $130{ }^{1}$

According to the latest WHO report, "one of the top 10 causes of death" is TB. Which is also the leading single infectious agent cause of death, ranking above human immunodeficiency virus (HIV)/acquired immunodeficiency syndrome. In 2018, HIV-negative patient mortality as a consequence of TB constituted approximately 1.2 million. ${ }^{1}$ A more concerning problem that arises with $\mathrm{TB}$ is the emergence of multidrugresistant (MDR) TB, which has increasing incidence. In Saudi Arabia, the current standard therapy for TB is rifampicin (RIF), isoniazid (INH), pyrazinamide (PZN), and ethambutol (EMB) for 2 months, followed by rifampicin and isoniazid for the remaining duration of treatment in pansensitive $\mathrm{TB}^{2}$ According to the $\mathrm{WHO}$ 2019 TB report: "There was some progress in testing and detection and treatment of MDR and RIF-resistant (RR) TB between 2017 and 2018. Globally, in 2018, $51 \%$ of individuals with bacteriologically confirmed TB were tested for RIF resistance, from $41 \%$ in 2017 ". ${ }^{1}$ The coverage of testing was $46 \%$ for new patients and $83 \%$ for previously treated patients with TB. A global total of 160,684 cases of MDR and RIF-resistant Mycobacterium TB complex (MTB) were reported in 2017. Of these, 139,114 cases started treatment. This number increased to 186,772 cases in 2018; of these, 156,071 cases started treatment. ${ }^{1}$ The incidence of MDR MTB in Saudi Arabia was approximately 6.7\%. However, estimations of the incidence of MDR MTB in our region vary in relation to the study period,

Disclosure. Authors have no conflict of interests, and the work was not supported or funded by any drug company. percentage of included non-Saudi patients, and specific study region. ${ }^{3}$ In addition, monodrug-resistant cases were shown to be more common than before. One crucial example is INH-monoresistant MTB. Its prevalence ranged from $2 \%$ to $>20 \%$ in previously untreated patients worldwide and averaged 10\% among all new cases. ${ }^{3}$ As shown in the WHO country profile for TB, the estimated incidence of TB in Saudi Arabia in 2018 is $3400(2,900-3,900)$ or $10(8.7-12)$ per 100,000 population, with an MDR/RR-TB incidence of 87 (64-110) or $0.3(0.2-0.3)$ per 100,000 population. Moreover, the proportion approximated among patients who possessed MDR or RR TB was 2.6\% (2-3.2) for new cases and 2.6\% (2-3.2) for previously treated cases. Data on INH monoresistance is limited. ${ }^{4}$

According to a previous retrospective study, which was conducted in Riyadh, Saudi Arabia, King Khalid University Hospital (KKUH), a total of 568 MTB cases were analyzed between January 2003 and December 2010 and showed monoresistance to INH (11\%), followed by $\operatorname{RIF}(7 \%)$ and $\operatorname{EMB}(2 \%){ }^{5}$ A study at King Abdulaziz University Hospital, Jeddah, Saudi Arabia, focused on investigating the trends of resistance to the current standard anti-TB regimen and revealed the monoresistance proportion to INH was $1 \%$, to PZN was $11.9 \%$, and to RIF was $2 \% .{ }^{6}$ Furthermore, RIF monoresistance is rare worldwide and is usually associated with MDR TB. ${ }^{7}$

Recent literature has shown INH monoresistance increased the likelihood of failure in treatments and relapse in patients treated with first-line anti-TB regimens. ${ }^{3}$ As $\mathrm{INH}$ is one of the main therapies used to treat latent tuberculosis infection (LTBI) in Saudi Arabia, and with a high prevalence proportion of $24 \%$ among healthcare workers, ${ }^{8}$ determining the prevalence of INH monoresistance is vital to know the nationwide substitution of INH with rifampin in the treatment of LTBI to help prevent further burden of TB. ${ }^{9}$ Determining the prevalence and the outcome of patients infected with monoresistant MTB compared with those with pansensitive MTB in KKUH were our primary objectives. This study can help reduce the knowledge gap in INH-monoresistant MTB and encourage further studies on this subject, which may guide further trends in resistance patterns for TB within the Middle East and help guide clinicians in the treatment of these cases. ${ }^{1}$

Methods. This is a cross-sectional study in which data were retrospectively collected from hospital records between May 2015 to April 2019 in KKUH, a tertiary care university hospital in Riyadh, Saudi Arabia. It provides outpatient, inpatient, cardiac, and intensive 
care services. Using Pubmed and Google Scholar, we searched for relevant published papers on our topic to conduct our literature review and utilized said papers in our discussion. The approval for this study was obtained from the university's Institutional Review Board (IRB) on December 8, 2019 (IRB number: E-19-4406). A total of 105 patients were included in this study. The inclusion criteria for this study was a positive culture for MTB between May 2015 and April 2019, while patients with positive mycobacteria cultures other than MTB were excluded. The following variables were collected from the patients' medical records: age at time of diagnosis, gender, residence, nationality, comorbidities, HIV status, site of infection, isolate, result of culture, date of admission, date of discharge, length of hospitalization, date of collection, date of treatment initiation, antibiotic resistance, level of INH monoresistance, treatment regimen, duration of treatment, previous treatment for $\mathrm{TB}$, treatment adverse events, and clinical outcomes. The important key definitions mentioned in the study are listed in Table 1.

For ethical reasons, all participants' confidentiality was ensured by assigning a code number to each participant solely for the purpose of research. Furthermore, patient files were not exchanged or disclosed to others outside of the research group.

Microbiological workup. Respiratory samples included in our study were sputum, bronchoalveolar lavage, endotracheal aspirate, and pleural fluid. Extrapulmonary samples included were tissue biopsy, sterile body fluids, cerebrospinal fluid (CSF), peritoneal fluid, pus, and gastric aspirates.
Nonsterile samples were decontaminated before processing. The samples were screened with microscopy by auramine-rhodamine and modified Ziehl-Neelsen stains and cultured in BACTEC ${ }^{\mathrm{TM}}$ MGIT $^{\mathrm{TM}} 960$ liquid medium (Becton Dickinson, New Jersey, United States) and Lowenstein-Jensen solid media. Samples were also processed with the Xpert MTB/RIF (Cepheid Inc., California, USA) assay for differentiation between MTB strains and nontuberculous mycobacteria and identification of RIF resistance through detection of mutation in the rpoB gene. Susceptibility testing was performed for the following anti-TB drugs: Streptomycin (STM), INH, RIF, EMB, and PZN using BACTEC $^{\text {TM }}$ MGIT $^{\text {TM }} 960$ SIRE and PZA Kits (Becton Dickinson, New Jersey, United States), following the manufacturer instructions. However, not all patients' samples underwent PZN susceptibility testing since it was not routinely available during the study period. In addition, the BACTEC $^{\mathrm{TM}}$ MGIT $^{\mathrm{TM}} 960$ SIRE Kit (Becton Dickinson, New Jersey, United States) was used to identify the resistance level of INH and was categorized into either high resistance $(0.4)$ or low resistance $(0.1)$.

Statistical analysis. Treatment regimens, patient characteristics, comorbidities, duration, and clinical outcomes were analyzed by SPSS version 25.0 statistical software for Mac OS, with a significance level determined in reference to a 2-tailed, type I error ( $p$-value) $<0.05$. Descriptive statistics (mean, median, standard deviation, frequencies, and percentages) were used to describe the quantitative and categorical variables. Also, logistical regression was carried out to assess if there is

Table 1 - Key definitions.

\begin{tabular}{ll}
\hline Terms & Definitions \\
\hline Cured & Patient with lab confirmed TB diagnosis with a negative smear or culture result in the last month of treatment \\
& with at least one more previous negative result. ${ }^{16}$ \\
Treatment completion & TB patient who completed their treatment without any indications or tests suggesting failure. ${ }^{17}$ \\
Treatment success & Patient with TB who met the criteria for "cured" or "treatment completion". \\
Treatment failure & TB patient with positive smear or culture after 5 months of treatment. ${ }^{16}$ \\
Death & TB patient who died during his treatment for any reason. ${ }^{16}$ \\
Relapse & Patients who were treated and labeled as "cured" or "treatment completed", and now present with a diagnosis \\
& of TB either relapsed or reinfected. ${ }^{16}$ \\
Previously treated & Patient with TB who has received any anti-TB medication. \\
Treatment unsuccess & Those who fulfill the following definitions: "treatment failure", "death", or "relapse" \\
Loss to follow-up & Patients with an interrupted course of treatment for at least two months. ${ }^{16}$ \\
Monodrug resistance & Resistance to a single TB medication only. ${ }^{16}$ \\
MDR Resistance & Patients with isolates resistant to both INH and RIF. ${ }^{16}$ \\
Resistance to INH & Growth of MTB was $>1 \%$ with $0.1 \mu \mathrm{g} / \mathrm{mL}$ (low) and $0.4 \mu \mathrm{g} / \mathrm{mL}$ (high)..$^{17,18}$ \\
\hline
\end{tabular}

TB: tuberculosis, INH: isoniazid, RIF: Rifampicin, MTB: Mycobacterium tuberculosis complex 
an association between patient comorbidities and INH monoresistance.

Results. A total of 105 patients were included in this study. The patterns of association were analyzed between those patients and different variables, such as age, gender, site of infection, and comorbidities. Table 2 describes the baseline characteristics of patients. Of 105 patients, 68 (64.8\%) were male, and $37(35.2 \%)$ were female. An overwhelming majority of patients were Saudi nationals ( $\mathrm{n}=85,81 \%$ ), while 19 were non-Saudis (18.1\%). A majority of patients were in the $21-40$ years age group $(\mathrm{n}=47,44.8 \%)$, followed by the $41-60$ years $(\mathrm{n}=25,23.8 \%)$, and $61-90$ years $(\mathrm{n}=24,22.9 \%)$ age groups, with a median age of 38 . Diabetes mellitus

Table 2 - Demographic and clinical characteristics of subjects $(\mathrm{N}=105)$.

\begin{tabular}{lc}
\hline Characteristics & $\mathbf{n}(\%)$ \\
\hline Age & \\
$1-20$ & $9(8.6)$ \\
$21-40$ & $47(44.8)$ \\
$41-60$ & $25(23.8)$ \\
$61-90$ & $24(22.9)$ \\
Mean & 42.93 \\
Median x-bar & 38.00 \\
Standard deviation & 21.329 \\
Gender & \\
Male & $68(64.8)$ \\
Female & $37(35.2)$ \\
Nationality & \\
Saudi & $85(81.0)$ \\
Non-Saudi & $19(18.1)$ \\
Not mentioned & $1(0.9)$ \\
Comorbidities & \\
Diabetes mellitus & $28(26.7)$ \\
Hypertension & $22(21.0)$ \\
Ischemic heart disease & $8(7.6)$ \\
Human immunodeficiency virus & $1(0.95)$ \\
Malignancy & $7(6.7)$ \\
Immunosuppression & $5(4.8)$ \\
Other comorbidities & $30(28.6)$ \\
No comorbidities & $50(47.6)$ \\
Previous treatment for tuberculosis & \\
Yes & $6(5.7)$ \\
No & $99(94.3)$ \\
Site of infection & \\
Pnknown & $6(5.7)$ \\
Extmonarapulmonary systems. HIV: Human immunodeficiency virus \\
Disseminated* \\
\hline
\end{tabular}

( $\mathrm{n}=28,26.7 \%)$ was the most common comorbidity in our patients, followed by hypertension $(n=22$, $21 \%)$, and ischemic heart disease $(\mathrm{n}=8,7.6 \%)$. Only one (1\%) patient with HIV was identified. Seven patients had malignancies $(6.7 \%)$ and 5 (4.8\%) who have been immunosuppressed by the use of biological therapy or pharmacotherapy. Other less common comorbidities were categorized together $(n=30,28.6 \%)$, such as bronchial asthma, atrial fibrillation, and hypothyroidism. Fifty (47.6\%) had no comorbidities. Most patients did not receive any previous treatments $(n=99,94.3 \%)$. We found that the most common type of infection was pulmonary $(n=39,37.1 \%)$, followed by extrapulmonary $(\mathrm{n}=34,32.4 \%)$, and disseminated infection $(n=26,24.8 \%)$. Six (5.7\%) patients had an unknown site of infection due to poor documentation.

Most patients ( $\mathrm{n}=91)$ were pansensitive to anti-TB medications, as shown in Table 3. Isoniazid was the most common antibiotic monoresistance $(n=9)$, followed by STM $(n=3)$. There was also one case of EMB resistance, and one case of MDR MTB. No patient had mono-RIF resistance or mono-PZN resistance or XDR-TB.

From our population of patients with INH monoresistance $(n=9), 7$ had low resistance levels. The age of patients with INH monoresistance was summarized in Table 4. The median age of patients with INH resistance was 40 . Moreover, most patients with INH monoresistance had disseminated TB. No patient with INH monoresistance had malignancies or had been immunosuppressed (Table 4).

The summary of treatment outcomes is listed in Table 5. After completing the course of treatment, a total of $56(53.3 \%)$ patients met the definition of successful treatment. Of the population with INH monoresistance, $5(55.6 \%)$ patients had a successful treatment outcome. Similarly, of the population with non-resistance, 51 $(53.1 \%)$ showed a successful treatment outcome.

Table 3 - Frequency and distribution of antibiotic resistance $(\mathrm{N}=105)$.

\begin{tabular}{lc}
\hline Antibiotic & $\mathbf{n}(\%)$ \\
\hline None & $91(86.7)$ \\
Isoniazid & $9(8.6)$ \\
Streptomycin & $3(2.9)$ \\
Ethambutol & $1(1.0)$ \\
MDR & $1(1.0)$ \\
Rifampicin & 0 \\
Pyrazinamide & 0 \\
\hline \multicolumn{2}{c}{ Values are presented as numbers and } \\
percentages (\%). MDT: multidrug-resistant \\
\hline
\end{tabular}


Table 4 - Frequency and distribution of isoniazid resistance (N=9).

\begin{tabular}{|c|c|c|}
\hline Variables & n (\%) & $P$-value \\
\hline \multicolumn{3}{|l|}{ Level of resistance } \\
\hline Low $(0.1)$ & $7(77.8)$ & \multirow[b]{2}{*}{ - } \\
\hline High $(0.4)$ & $2(22.2)$ & \\
\hline \multicolumn{3}{|l|}{ Age } \\
\hline $1-20$ & 0 & \multirow{7}{*}{0.774} \\
\hline $21-40$ & $5(55.5)$ & \\
\hline $41-60$ & $2(22.2)$ & \\
\hline $61-90$ & $2(22.2)$ & \\
\hline Mean x-bar & 42.44 & \\
\hline Median & 40.00 & \\
\hline Standard deviation & 19.951 & \\
\hline \multicolumn{3}{|l|}{ Site of infection } \\
\hline Pulmonary & $2(22.2)$ & \multirow{3}{*}{0.400} \\
\hline Extrapulmonary & $2(22.2)$ & \\
\hline Disseminated & $4(44.4)$ & \\
\hline \multicolumn{3}{|l|}{ Gender } \\
\hline Male & $6(66.6)$ & \multirow[t]{2}{*}{0.900} \\
\hline Female & $3(33.3)$ & \\
\hline \multicolumn{3}{|l|}{ Comorbidities } \\
\hline Diabetes mellitus & $2(22.2)$ & 0.753 \\
\hline Hypertension & $3(33.3)$ & 0.340 \\
\hline Ischemic heart disease & 0 & 0.368 \\
\hline HIV & 0 & 0.758 \\
\hline Malignancy & 0 & 0.402 \\
\hline Immunosuppression & 0 & 0.483 \\
\hline Other comorbidities & $3(33.3)$ & 0.741 \\
\hline No comorbidities & $3(33.3)$ & 0.231 \\
\hline $\begin{array}{r}\text { Values are presen } \\
\text { HIV: Hu }\end{array}$ & $\begin{array}{l}\text { Imbers an } \\
\text { nunodefic }\end{array}$ & (\%). \\
\hline
\end{tabular}

A total of $13(12.4 \%)$ patients met the definition of unsuccessful treatment. Of the population with INH monoresistance, one patient showed an unsuccessful treatment outcome with that outcome being death. Twelve (12.5\%) patients with nonresistance also showed unsuccessful treatment outcomes. Eight with no resistance died and 4 had relapses. Moreover, a total of $36(34.3 \%)$ patients had no follow-up records, with $3(33.3 \%)$ in the INH-monoresistant group, and 33 (34.4\%) patients with nonresistance. The duration of treatment in months is also summarized in Table 5. The resistant group had 3 patients who had a 7-9 month treatment duration, 2 patients with 10-12 months, and one patient with $>12$ months of treatment. Alternatively, the majority of the nonresistant group had 7-9 months of treatment. However, in contrast to the resistant group, 15 patients had 1-6 months of treatment, 9 patients had 10-12 months of treatment, and 7 patients had $>12$ months of treatment. Furthermore, the resistant group has a mean treatment duration of 12 months with a standard deviation of 6 , while the nonresistant group
Table 5 - Outcomes between patients with isoniazid-monoresistant and those with non-isoniazid resistance $(\mathrm{n}=105)$.

\begin{tabular}{|c|c|c|c|}
\hline Outcome & $(n=9)$ & $(n=96)$ & $P$-value \\
\hline $\begin{array}{l}\text { Duration of treatment } \\
\text { (months) }\end{array}$ & Resistant & Nonresistant & \multirow{8}{*}{0.581} \\
\hline $1-6$ & 0 & $15(16.3)$ & \\
\hline $7-9$ & $3(3.3)$ & $32(34.7)$ & \\
\hline $10-12$ & $2(22.2)$ & $9(9.2)$ & \\
\hline$>12$ & $1(11.1)$ & $7(6.1)$ & \\
\hline Loss to follow-up & $3(33.3)$ & $33(33.3)$ & \\
\hline $\begin{array}{l}\text { Mean x-bar (excluding } \\
\text { loss to follow-up) }\end{array}$ & 12.00 & 9.46 & \\
\hline Standard deviation & 6.033 & 3.711 & \\
\hline Side effects & Resistant & Nonresistant & \multirow{7}{*}{0.079} \\
\hline Drug-induced hepatitis & $1(11.1)$ & $12(12.2)$ & \\
\hline Blurry vision & $1(11.1)$ & $1(1.02)$ & \\
\hline Peripheral neuropathy & $1(11.1)$ & $1(1.02)$ & \\
\hline Joint pain & 0 & $4(4.1)$ & \\
\hline No side effects & $6(66.67)$ & $71(72.5)$ & \\
\hline Other side effects & 0 & $9(9.2)$ & \\
\hline Clinical outcome & Resistant & Nonresistant & \multirow{7}{*}{0.988} \\
\hline Successful treatment & $5(55.56)$ & $51(53.13)$ & \\
\hline Unsuccessful treatment & $1(11.11)$ & $12(12.5)$ & \\
\hline$\rightarrow$ Relapse & 0 & 4 & \\
\hline$\rightarrow$ Death & 1 & 8 & \\
\hline Loss to follow-up & $3(33.33)$ & $33(34.38)$ & \\
\hline $\begin{array}{l}\text { Length of hospitalization } \\
\text { (days) }\end{array}$ & Resistant & Nonresistant & \\
\hline $1-25$ & 1 & 43 & \multirow{6}{*}{0.130} \\
\hline $26-50$ & 2 & 20 & \\
\hline$>50$ & 1 & 11 & \\
\hline Unavailability & 5 & 22 & \\
\hline $\begin{array}{l}\text { Mean x-bar (excluding } \\
\text { loss to follow-up) }\end{array}$ & 38.00 & 36.96 & \\
\hline Standard deviation & 20.314 & 53.475 & \\
\hline
\end{tabular}

Values are presented as numbers and percentages (\%).

had a significantly shorter treatment duration, with 9.5 months and a standard deviation of 3.7.

As for adverse events, Table 5 summarized the INHmonoresistant group. One patient was positive for HIV and had no resistance to any anti-TB medication. However, the patient died after a long treatment duration that exceeded 12 months. Binary logistic regression was carried out to assess the association between comorbidities and INH-monoresistance, and no statistically significant association was found.

Finally, the changes of INH-resistant and nonresistant cases throughout the study period can be visualized in Figure 1.

Discussion. In our study, we found that the prevalence of INH-monoresistant MTB was $8.6 \%$ $(n=9)$. Our findings are similar to the international 
prevalence of INH monoresistance $(8.6 \%$ vs $7.4 \%) .{ }^{10}$ However, our prevalence was lower compared to previous local studies ( $8.6 \%$ vs $10 \%)$. A potential reason for this finding is the small sample size, compared to the larger sample pool of 2015, in the systematic review study that estimated prevalence proportion of $10.13 \%$ for INH monoresistance. ${ }^{11}$ Another potential reason is the improvements in the quality of the healthcare system and accumulated knowledge for the treatment of TB. Moreover, we found that our prevalence proportion is lower than those in other regions in Saudi Arabia such as in Jeddah $(8.6 \%$ vs $28.7 \%),{ }^{12}$ Jizan $(8.6 \%$ vs $41 \%),{ }^{13}$ and the Eastern region $(8.6 \%$ vs $9.8 \%) ;{ }^{14}$ but, higher than the prevalence proportion in Taif $(8.6 \%$ vs $6.5 \%) .{ }^{13}$

The treatment outcome for patients with INH monoresistance was highly variable with other study results. ${ }^{3}$ Previous studies suggested that INH monoresistance increases the likelihood of treatment failure. ${ }^{3}$ However, in this study, both patients with INH monoresistance and patients with nonresistance had the same percentage of unsuccessful treatment. The length of hospitalization was longer in patients with nonresistance, which also conflicts with other studies. Moreover, the majority of side effects were noted in nonresistant cases. However, longer treatment duration was observed for patients with INH monoresistance, which is consistent with other studies. ${ }^{3}$

Treatment regimens were adjusted based on adverse effects and physicians' preferences. Regimens were also frequently extended. We had a total of 28 (26.7\%) patients who had side effects. Contrary to other studies, the majority of side effects were noted in nonresistant cases as there were $25(26 \%)$ patients with non-INH monoresistance who had side effects compared to only $3(33.3 \%)$ patients with INH monoresistance. Similar to other reports, hepatotoxicity was common among patients. ${ }^{3}$ The resistant group had one $(11.1 \%)$ patient, while the nonresistant group had $12(12.2 \%)$ patients who had drug-induced hepatitis.

Similar to other studies, the treatment duration varied between patients with INH monoresistance, with the majority of patients having a treatment duration ranging between 7 and 12 months. ${ }^{15}$

Moreover, this study showed that patients with drug-susceptible MTB could have an adverse outcome similar to patients with INH monoresistance.

Study limitation. A significant limitation to this study was noted in the data collection process. The electronic health record had poor documentation. Additionally, our inability to control confounding variables, such as age and comorbidities, for assessing patients' outcomes. Furthermore, not all patients underwent PZN susceptibility testing due to the limited availability of testing kits. Although our study included a small number of patients with INH monoresistance, with KKUH being a large tertiary care center receiving multiple patients from various regions with similar genetic and geographical backgrounds, the results from this study can be generalized to other centers in the region.

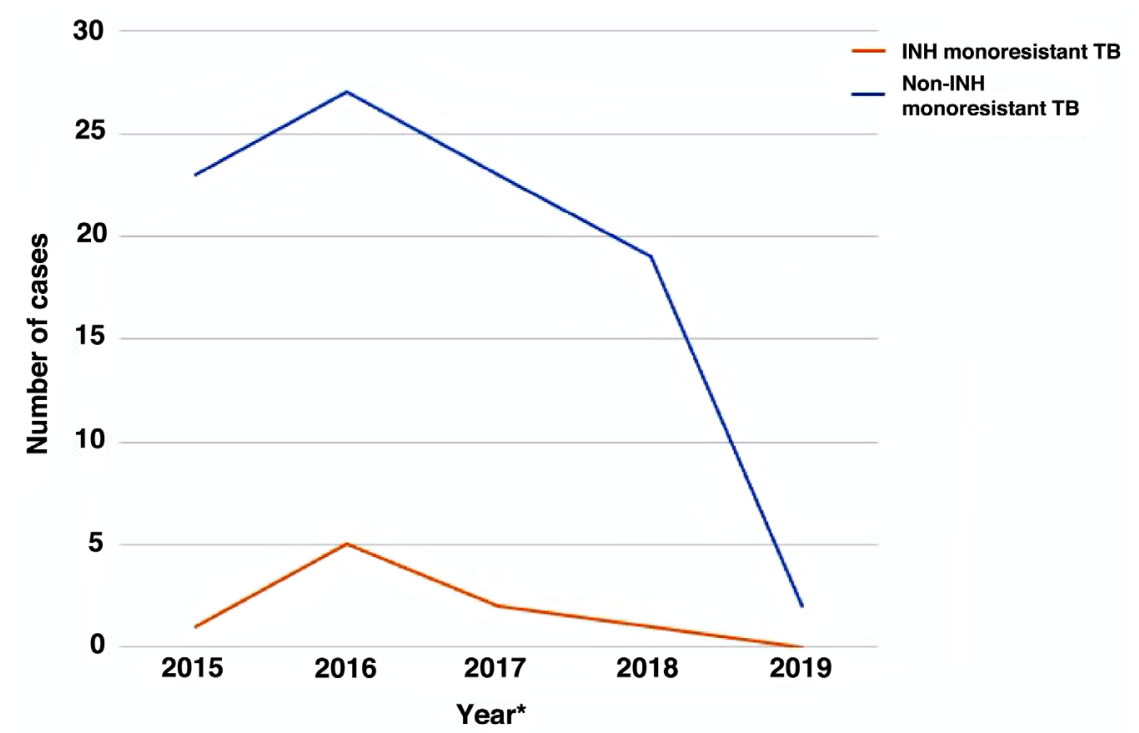

Figure 1 - *Samples collected from 1st of May 2015 to 30th of April 2019. INH: isoniazid, TB: tuberculosis 
The implications explored in this study can help future regional and global tuberculosis surveillance studies. Moreover, this study can be used in future systematic reviews to assess the use of anti-TB medications with INH monoresistance and treatment outcome and their treatment outcome.

In conclusion, INH monoresistance is the most prevalent type of resistance to medications for the anti-TB regimen found in our population. Our study has not shown any substantial significance in the outcome variant compared to nonresistant cases. This observation may be attributable to our population's limited number of INH-monoresistant cases. A more extensive study with abundant INH-monoresistant cases is required to establish further association between the level of INH monoresistance and outcome.

Acknowledgment. The authors want to express their gratitude to the Deanship of Scientific Research and RSSU, King Saud University, Riyadh, Saudi Arabia for their technical support. The author also acknowledge Enago (www.enago.com) for English language editing.

\section{References}

1. World Health Organization. Global tuberculosis report 2019. [Internet] Geneva (SW): World Health Organization; [Updated 2019. Cited 2020 Sept 24]. Available from: https://www.who. int/publications/i/item/global-tuberculosis-report-2019

2. Metry AM, Al Salmi I, Al-Abri S, Al Ismaili F, Al Mahrouqi Y, Hola A, et al. Epidemiology and outcome of tuberculosis in immunocompromised patients. Saudi J Kidney Dis Transpl 2017; 28: 806-817.

3. Gegia M, Winters N, Benedetti A, van Soolingen D, Menzies D. Treatment of isoniazid-resistant tuberculosis with first-line drugs: a systematic review and meta-analysis. Lancet Infect Dis 2017; 17: 223-234.

4. World Health Organization. Tuberculosis Country Profiles online tool. [Updated 2019. Cited 2020 Sept 24]. Available from: https://worldhealthorg.shinyapps.io/tb_profiles/?_inputs \&lan=\%22EN\%22\&iso2=\%22SA\%22

5. Somily AM, Naeem T, Habib HA, Sarwar MS, Kunimoto DY, Kambal AM. Changing epidemiology of tuberculosis detected by an 8-year retrospective laboratory study in a tertiary teaching hospital in central Saudi Arabia. Saudi Med J 2014; 35: 691-698.

6. Jiman-Fatani A, El-Hossary D, Eltahlawi RA. Mycobacterium tuberculosis complex: Detection and patterns of resistance to the first line anti-TB drugs at the King Abdulaziz University Hospital, Saudi Arabia. IAJAA 2016; 5: 2.
7. Gibson J, Donnan E, Eather G. Management of rifampicin mono-resistant tuberculosis in Queensland, Australia: a retrospective case series. Respirol Case Rep 2018; 6: e00366.

8. Almohaya A, Aldrees A, Akkielah L, Hashim AT, Almajid F, Binmoammar $\mathrm{T}$, et al. Latent tuberculosis infection among health-care workers using Quantiferon-TB gold-plus in a country with a low burden for tuberculosis: prevalence and risk factors. Ann Saudi Med 2020; 40: 191-199.

9. Menzies D, Adjobimey M, Ruslami R, Trajman A, Sow O, Kim $\mathrm{H}$, et al. Four months of rifampin or nine months of isoniazid for latent tuberculosis in adults. N Engl J Med 2018; 379: 440-453.

10. Dean AS, Zignol M, Cabibbe AM, Falzon D, Glaziou P, Cirillo DM, et al. Prevalence and genetic profiles of isoniazid resistance in tuberculosis patients: A multicountry analysis of cross-sectional data. PLoS medicine 2020; 17: e1003008.

11. Al-Tawfiq JA, Hinedi K, Memish ZA. Systematic review of the prevalence of Mycobacterium tuberculosis resistance in Saudi Arabia. J Chemother 2015; 27: 378-382.

12. Khan MY, Kinsara AJ, Osoba AO, Wali S, Samman Y, Memish Z. Increasing antimicrobial resistance of M. tb in a Saudi Arabia referral hospital. Int J Antimicrob Agents 2001; 17: 415-418.

13. Abu-Amero KK. Status of antituberculosis drug resistance in Saudi Arabia 1979-98. East Mediterr Health J 2002; 8: 664-670.

14. Chaudhry LA, Rambhala N, Al-Shammri AS, Al-Tawfiq JA. Patterns of antituberculous drug resistance in eastern Saudi Arabia: a 7-year surveillance study from 1/2003 to 6/2010. J Epidemiol Glob Health 2012; 2: 57-60.

15. Cattamanchi A, Dantes RB, Metcalfe JZ, Jarlsberg LG, Grinsdale J, Kawamura LM, et al. Clinical characteristics and treatment outcomes of patients with isoniazid-monoresistant tuberculosis. Clin Infect Dis 2009; 48: 179-185.

16. World Health Organization. Definitions and reporting framework for tuberculosis-2013 revision. [Updated 2021. Cited 2020 Sept 24]. Available from: https:/www.who.int/tb/ publications/definitions/en/

17. Woods GL, Brown-Elliott BA, Conville PS, Desmond EP, Hall GS, Lin G, et al. Susceptibility Testing of Mycobacteria, Nocardiae, and Other Aerobic Actinomycetes. 2nd ed. Wayne (PA): Clinical and Laboratory Standards Institute; March 2011.

18. Becton BD. Dickinson and Company Newsletter BD Bactec MGIT 960 SIRE kit now FDA-cleared for susceptibility testing of Mycobacterium tuberculosis. Microbiology News \& Ideas. (Updated 2016. Updated 2016. Cited 2020 Feb 5) Available from URL: https://legacy.bd.com/ds/technicalCenter/ inserts/8008200(04).pdf 